\title{
Л.И. Соболевская
}

\section{Подход к интеллектуализации системы поддержки принятия решений при оценке и анализе состояния предприятий растениеводства}

\author{
Обоснована целесообразность построения и использования байесовской сети доверия как экспертной системы при исследова- \\ нии экономической эффективности функционирования группы предприятий отрасли растениеводства в регионе. \\ Ключевые слова: аграрный сектор, экспертные системы, байесовский подход, байесовские сети. \\ Обгрунтовано доцільність побудови та використання байєсівської мережі довіри як експертної системи при дослідженні еко- \\ номічної ефективності функціонування групи підприємств рослинницької галузі регіону. \\ Ключові слова: аграрний сектор, експертні ситстеми, байєсівській підхід, байєсівські мережі.
}

Введение. Значительное влияние на стабильное функционирование аграрного сектора и его отдельных отраслей имеет учет и корректировка действий внешних и внутренних факторов. Для аграрного сектора экономики, непосредственно связанного с использованием природных ресурсов, этот фактор имеет особое значение. Разработка технологических средств интеллектуального моделирования социальноэкономических систем базируется на структуризации знаний о внутренних и внешних факторах и условиях устойчивого развития социально-экономических систем.

Интеллектуализация процессов принятия решений в рамках современных информационных систем управления способствует повышению эффективности управленческих решений, помогает менеджерам бизнес-процессов принимать верные, своевременные эффективные решения, что актуально в современных условиях. Состояние современной бизнес-среды практически в любой отрасли характеризуется непрерывным усложнением, высокими темпами развития, снижением предсказуемости и требованиями обеспечения эффективного и устойчивого функционирования.

В общем случае интеллектуализация процессов принятия решений предусматривает предоставление информационным системам управления следующих дополнительных возможностей [1]:

- планирование цепи перехода от существующего состояния к желаемому результату при заданной общесистемной цели функционирования;
- оценка информации по степени важности и соответствующая ее сортировка при заданных критериях отбора информации;

- поиск управленческих решений в условиях неполной и нечеткой информации при использовании заданных эвристик и опыта экспертов предметной области;

- оценка результативности полученных управленческих решений и определение условий достижения данного эффекта;

- изменение стратегии функционирования системы при изменении характера решаемых задач;

- формирование, пополнение и корректировка базы знаний.

Экспертные системы (ЭС) рассматриваются как разновидность системы поддержки принятия решений (СППР), позволяющие использовать в процессе работы накопленные специалистами опыт и знания и в свою очередь генерируют новые знания, стимулирующие развитие исследуемой экономической системы.

\section{Анализ последних исследований}

В работах [1-6] рассматриваются вопросы, связанные с процессами интеллектуализации информационных СППР для оценки и анализа состояний малого предприятия. Разработка данных систем предусматривает для пользователя возможность получения информации на основе обработки данных и использования в процессе работы полученной информации и накопленный профессионалами опыт и знания. Под информационной СППР понимается любая компьютерная информационная система, 
которая предоставляет при принятии решений помощь различного характера (вычисление, поиск, формирование вывода, удобное представление результатов и т.д.). Как разновидность СППР рассматриваются ЭС [2, 4]. В [2] выделены шесть этапов технологии разработки ЭС:

- идентификация: определение задач, подлежащих решению, установление целей разработки, определение экспертов и типов пользователей;

- концептулизация: содержательный анализ проблемной области, выявление имеющихся понятий и их взаимосвязей, определение методов решения поставленных задач;

- формализация: выбор инструментария, определение способов представления всех типов знаний. Формализация основных понятий, определение способов интерпретации знаний, моделирование работы системы, оценка адекватности понятий, методов решения, средств представления и манипулирования знаниями;

- наполнение экспертом базы знаний;

- тестирование;

- эксплуатация.

Процесс накопления знаний разделяют на получение знаний от эксперта, организацию знаний, обеспечивающую эффективную работу системы, и представление знаний в виде понятном ЭС.

Как возможные подходы к разработке ЭС выделяют следующие:

- системы на основе правил;

- системы с использованием нейронных сетей;

- экспертные системы на основе сетей доверия Байеса (БС).

Выбирая подход при создании ЭС для исследуемой экономической системы, необходимо выделить такой класс задач из возможного множества, который актуален для данной системы и качественно решается с помощью данного инструментария.

Цель статьи - определение основных принципов разработки знание-ориентированной модели с учетом факторов устойчивого развития отрасли растениеводства.

\section{Постановка задачи}

Необходимо обосновать целесообразные подходы и определить принципы построения соответствующей ЭС при исследовании факторов устойчивого функционирования предприятий отрасли растениеводства.

\section{Методика управления развитием агро- предприятий}

Как экономическую систему рассматриваем определенную группу предприятий отрасли растениеводства, объединенных производством и переработкой ее продукции.

Актуальным считаем разработку подходов к исследованию экономической устойчивости аграрных предприятий, что в свою очередь предусматривает осуществление эффективного управления такими структурными составляющими, как финансовая, производственная, организационно-кадровая, инвестиционно-инновационная, функционирование которых осуществляется в условиях неопределенности и риска.

При разработке ЄС для данной группы предприятий отрасли в качестве одной из основных можно выделить задачу стратегического анализа результатов и последствий реализации различных вариантов развития группы предприятий отрасли растениеводства в условиях неопределенности и риска, соответствующих различным вариантам внедрения инновационноинвестиционных технологий.

Управление развитием аграрного сектора предусматривает оптимизацию внедрения инвестиционно-инновационных проектов на основе частно-государственного партнерства, в том числе государственного кредитования.

Данная задача предполагает распределение средств на региональном уровне между секторами агропромышленного комплекса (АПК) в зависимости от индикаторов, характеризующих состояние секторов. Также предполагается поэтапная оптимизация инвестиционных средств между группами предприятий отрасли растениеводства в исследуемом регионе [7].

На первом этапе определяем возможные варианты поэтапного кредитования $\left(j_{t} € J_{t}\right)$ инвестиционно-инновационных проектов данных $L$ групп предприятий. 
В качестве критериев оптимизации могут быть рассмотрены:

- минимизация затрат, связанных с внедрением проекта;

- критерии доходности данного проекта.

Основными ограничениями можно считать:

- ограничение по возможным кредитным заимствованиям в год $t: K\left(j_{t-1}, j_{t}\right) \chi\left(j_{t-1}, j_{t}\right) \times$ $\times \chi^{*}\left(j_{t}\right) \leq k_{t}$,

- ограничение по возможным значениям риска кредитоспособности предприятия в год $t$ : $r\left(j_{t}\right) \leq R_{t}$.

Задача второго этапа базируется на результатах решения задачи первого этапа и может быть сформулирована как задача оптимального распределения на региональном уровне инвестиционных ресурсов между группами предприятий растениеводческой отрасли региона.

Алгоритм математического моделирования, используемый для решения данной задачи, пошаговое построение и оптимизация вариантов решения, основанный на методе последовательного анализа вариантов. Данный метод предусматривает разработку и обоснование правил пошагового построения, сравнения и отбраковки вариантов, которые при дальнейшем их развитии не могут войти в состав оптимального решения [8].

Управление устойчивым развитием аграрного сектора предполагает также одновременно разработку и исследование показателей, критериев и индикаторов устойчивого функционирования аграрного сектора (на уровне региона, отрасли, предприятий). Схематическая модель построения системы управления экономической эффективностью сельскохозяйственных предприятий, специализирующихся на выращивании овощей или злаков, приведена на рис. 1.

При исследовании экономической эффективности функционирования группы предприятий растениеводческой отрасли региона, объединенных производством и переработкой ее продукции, целесообразным можно считать построение и использование байесовской сети доверия (БСД) как ЭС. Определен подход к установле-

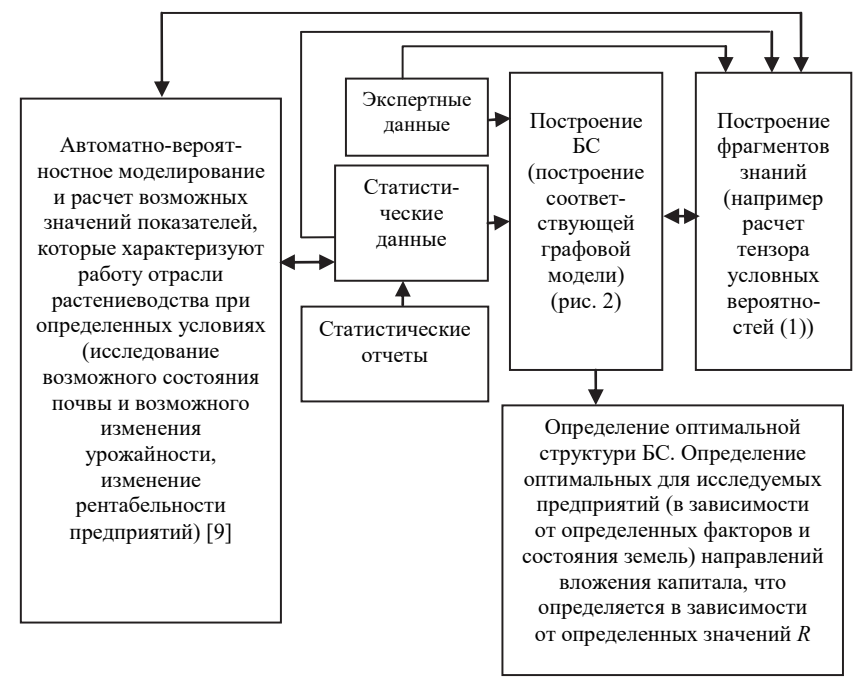

Рис. 1. Схематическая модель построения системы управления экономической эффективностью сельскохозяйственных предприятий

нию рейтинга группы предприятий отрасли на основе разработки системы показателей, характеризующих функционирование предприятий исследуемой отрасли и установление нормативных значений каждого показателя по классам финансового состояния в соответствии с практикой анализа предприятий данной отрасли. Возможные изменения отдельных показателей в нормативных пределах будут определять множество альтернатив функционирования группы предприятий отрасли - возможные состояния исследуемой системы (множество значений показателя $R$ ). Подход к исследованию экономической эффективности функционирования, рентабельности $(R)$ и других показателей $\left(F_{i}\right)$, связанных с ними, для определенной группы сельскохозяйственных предприятий отдельного региона, специализирующихся на выращивании и переработке растений конкретного типа, и осуществление их классификации (ранжирование) по уровню значений рентабельности на определенном интервале функционирования заключается в следующем. Интервалы возможных значений рентабельности $(R)$ разобьем на четыре группы устойчивости: высокой, удовлетворительной, низкой и критической.

Считаем целесообразным осуществлять ранжирование хозяйств растениеводческой отрасли по уровню значений показателей $R$, используя подход к исследованию системы основных пока- 
зателей и факторов $\left(F_{i}\right)$, которые обусловливают значение $R$, основанный на построении и использовании байесовских сетей (рис. 2). БС состоит из множества узлов и совокупности направленных ребер, соединяющих эти узлы между собой. Ребра определяют причинно-следственные связи в предметной области, которые в подавляющей мере не есть однозначно определенными. Правдоподобность утверждения задается с помощью вероятности. Концепция БС заключается в обновлении вероятностей при поступлении дополнительной информации и базируется на теореме Байеса (формула (1)):

$$
P\left(R / F_{1}, F_{2}, \ldots, F_{n}\right)=\frac{P(R) \prod_{i=1}^{n} P\left(F_{i} / R\right)}{P\left(F_{1}, \ldots, F_{n}\right)},
$$

где $F_{i}$ - реализация $i$-й из $n$ выбранных переменных; $R$ - рентабельность предприятия.

Суть обучения БС заключается в корректировке вероятностей при получении новой информации о совокупности состояний ее узлов.

Данный подход лежит в основе методологии ранжирования предприятий отрасли растениеводства по значениям показателей, характеризующих экономическую устойчивость отрасли. Рассмотрим построение БС для определения влияния существенных показателей и факторов, связанных с производством продукции в отрасли растениеводства, на рентабельность (для сельскохозяйственных предприятий).

Систематизируем вершины (показатели и критерии) БС (рис. 2):

- производительность труда - ПТ; объем производства - ОП;

• колебания цен - КЦ; ожидаемый спрос на продукцию - ОС;

- погодные условия - ПУ; уровень загрязнения земель - УЗ3;

- уровень производительности агроландшафта $-S$; урожайность - У;

- объем капиталовложений - ОК; производственные затраты - П3; финансовый результат (рентабельность) - ФР.

Для каждой вершины сети необходимо определить множество возможных состояний [10].

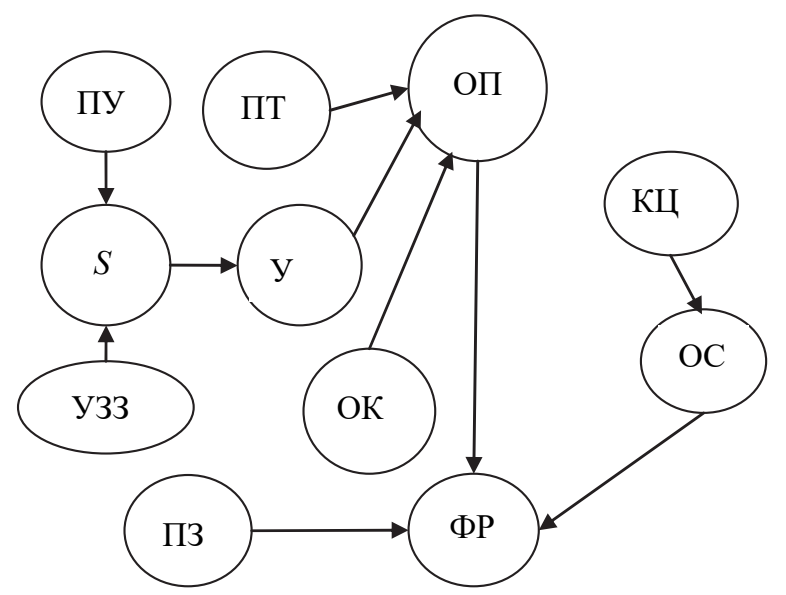

Рис. 2. БС для определения влияния показателей и факторов на рентабельность

Описание вершины (узлов) БС и их возможных состояний приведено в табл. 1.

Таким образом выделены факторы и условия устойчивости аграрного сектора, которые определяются значениями соответствующих факторов и показателей.

Последним и самым важным шагом при построении БС необходимо построение таблиц условных вероятностей (ТУВ) для каждой вершины, построение которой осуществляется в соответствии с формулой Байеса (1). На основе значений ТУВ строится ЭС, от точности ее значений зависит адекватность построенной модели. ТУВ, созданные на основе полученной от экспертов информации, должны обеспечивать выполнение логических взаимосвязей, характерных для предметной области. Структурная схема корректировки базы знаний $($ табл. 1,2 ) представлена на рис. 3 .

Отметим, что расчет вероятностей значений показателей экономической эффективности функционирования агропредприятий может осуществляться и в случае возможного применения различных стратегий (внедрение соответствующих инвестиционных технологий), оказывающих влияние на интенсификацию производства и связанных с определенными необходимыми затратами. Основное ограничение при внедрении определенного варианта технологии максимально возможный объем капиталовложений $\left(\mathrm{OK}_{t}^{\max }\right)$ в $t$-й год внедрения технологии и выполнение условия для показателей ОС и ОП: $\mathrm{O} \Pi \mathrm{OC}$. 
T а б л и ц а 1. Совокупность узлов БС, их возможные состояния и стратегическое значение

\begin{tabular}{|c|c|c|}
\hline Вершина & Возможные состояния & Характеристика вершин \\
\hline $\begin{array}{l}\text { Ожидаемый спрос на про- } \\
\text { дукцию растениеводства }\end{array}$ & $\begin{array}{l}\text { Рост } \\
\text { Снижение }\end{array}$ & $\begin{array}{l}\text { Увеличение спроса ведет к уменьшению излишков и } \\
\text { потенциальному росту доходов }\end{array}$ \\
\hline $\begin{array}{l}\text { Колебания цен на внутрен- } \\
\text { нем рынке }\end{array}$ & $\begin{array}{l}\text { Ценовая стабильность } \\
\text { Допустимое снижение } \\
\text { Критическое снижение }\end{array}$ & $\begin{array}{l}\text { Стабильность цен на внутреннем рынке обеспечива- } \\
\text { ется путем стабилизации внутреннего предложения } \\
\text { на основе регулирования объемов производства }\end{array}$ \\
\hline $\begin{array}{l}\text { Объемы капиталовложений } \\
\text { по группе предприятий }\end{array}$ & $\begin{array}{l}\text { Оптимальные (рассчитанные по опти- } \\
\text { мизационной модели) } \\
\text { Допустимо ниже оптимальных } \\
\text { Низкие }\end{array}$ & $\begin{array}{l}\text { При государственной поддержке внедрение новых } \\
\text { технологий будет способствовать росту прибыльно- } \\
\text { сти и рентабельности }\end{array}$ \\
\hline Погодные условия & $\begin{array}{l}\text { Благоприятные } \\
\text { Нейтральные } \\
\text { Неблагоприятные }\end{array}$ & $\begin{array}{l}\text { Влияет на развитие производства определенных ви- } \\
\text { дов продукции }\end{array}$ \\
\hline Уровень загрязнения земель & $\begin{array}{l}\text { Низкий } \\
\text { Средний } \\
\text { Высокий }\end{array}$ & $\begin{array}{l}\text { Вместе с погодными условиями влияет на уровень } \\
\text { производительности агроландшафта }\end{array}$ \\
\hline Производственные затраты & $\begin{array}{l}\text { Низкие } \\
\text { Средние } \\
\text { Высокие }\end{array}$ & $\begin{array}{l}\text { Соответствуют затратам по производству и перера- } \\
\text { ботке продукции }\end{array}$ \\
\hline Рентабельность & $\begin{array}{l}\text { Интервалы возможных значений по- } \\
\text { казателя } R \text { : } \\
\text { высокая } \\
\text { удовлетворительная } \\
\text { низкая } \\
\text { критическая }\end{array}$ & $\begin{array}{l}\text { Отношение эффекта к затратам предпринимательской } \\
\text { деятельности }\end{array}$ \\
\hline
\end{tabular}

Для текущей стратегии развития исследуемой группы предприятий отрасли растениеводства строим БС (рис. 2)

$$
\begin{aligned}
& \hline \\
& \hline \\
& \hline \\
& \hline
\end{aligned}
$$

Построение базы знаний на основе фрагментов знаний (расчет тензора условных вероятностей (1))

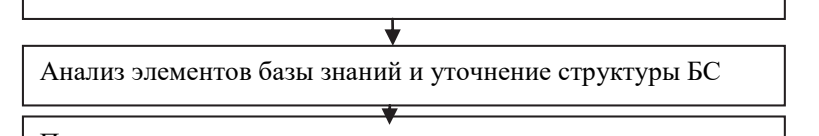

Проверка возможного изменения внешних и внутренних Изменение априорных вероятностей для корневых вершин БС, что соответствует изменению определенных факторов. Перерасчет значений определенных показателей по соответствующей автоматной модели

Корректирование базы знаний

\begin{tabular}{|l|}
\hline \multicolumn{1}{|c|}{$\begin{array}{l}\text { Оценка привлекательности текущей стратегии развития } \\
\text { исследуемой группы предприятий отрасли растениеводства и } \\
\text { сравнение ее с предыдущей }\end{array}$} \\
\hline
\end{tabular}

Формирование следующей возможной стратегической альтернативы.

Рис. 3. Структурная схема корректировки базы знаний
Рассмотрим упрощенный пример применения предложенного подхода к оценке экономической эффективности предприятий отрасли растениеводства. Предположим, что данная группа предприятий специализируется на выращивании определенного типа зерна и его обработке на зерносушильных установках.

Общая задача нахождения условных вероятностей для соответствующих состояний узлов БС, которые используются для определения влияния показателей и факторов на рентабельность (ФР) данной группы предприятий, может быть представлена как задача оновления вероятностей на подсетях, в состав которых входят лишь определенные подмножества узлов графа. Исследование такого показателя, как урожайность, осуществляется с помощью байесовской подсети (рис. 4). Исследование производственных затрат, связанных с использованием зерносушильных установок определенного типа, представлено как анализ возможных значений узлов соответствующей подсети (рис. 5). Ана- 
лиз $R$ осуществляется на основе расчета возможных значений ее вероятности при определенных реализациях показателей У и $V$ (рис. 6).

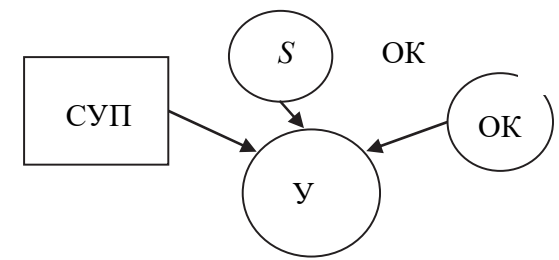

Рис. 4. Исследование показателя У (урожайность)

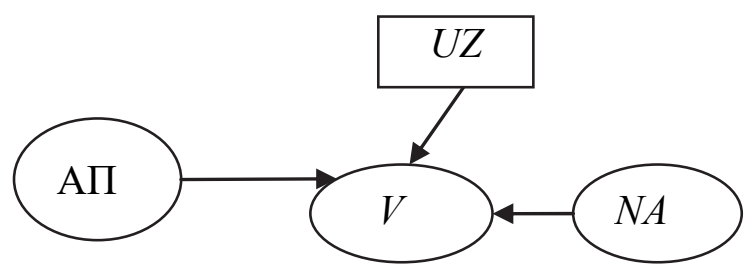

Рис. 5. Исследование показателя $V$ - производственные затраты, связанные с использованием зерносушильной машины определенного типа

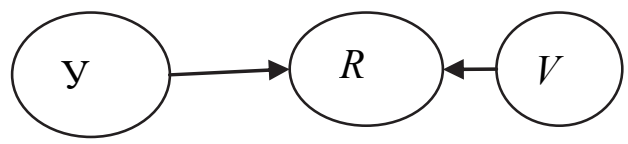

Рис. 6. Анализ возможных состояний рентабельности

Используем такие обозначения: У - урожайность; СУП - уровень снижения урожайности из-за погодных условий; $S$ - уровень продуктивности агроландшафта (отношение фактической продуктивности (урожайности) сельскохозяйственных культур к потенциальной); ОК - объем капиталовложений; $V$ - затраты, связанные с просушкой зерна; $N A$ - норма амортизации оборудования; АП - арендная плата за размещение зерносушильных машин; $U Z$ - уровень загрузки оборудования.

Таким образом целесообразным может быть использование метода кластеризации, когда в роли вершин выступают не отдельные вершины, а их группы (кластеры).

На рис. 4, 5, 6 вершины, принимающие дискретные значения, обозначены квадратом. Вершины, изображенные в виде круга, соответствуют непрерывным случайным величинам. Считаем, что исследуемые непрерывные величины имеют распределение Гаусса, т.е., если вершине $X$ соответствуют родительские вершины $U=\left\{U_{1}, U_{2}, \ldots, U_{n}\right\}$, то условное распределение вероятностей для $X$ определяется по формуле:
$f\left(X / U_{i}\right)=N\left(x ; \mu_{x}++b_{i} \times \mu_{\mathrm{i}}, \sigma_{x}\right)$, где $\mu_{x}$ и $\mu_{i}-$ математические ожидания случайных величин, которые соответствуют вершинам $x$ и $i ; \sigma_{x}-$ дисперсия соответствующей случайной величины; $b_{i}$ - определяет связь между $X$ и ее родительской вершиной $i$ (весовой коэффициент влияния).

В общем случае будем обозначать дискретные случайные величины, соответствующие узлам графа, через $X_{j}(j=1, \ldots, s)$. Каждая из этих случайных величин имеет своими возможными реализациями значения $x_{i}(i=1, \ldots, n)$ с вероятностями $P_{i j}$, для которых $\sum_{i=1}^{n j} P_{i j}=1$. Совместное влияние дискретных случайных величин на вершину $Y$ характеризуется математическими ожиданиями $\left(\mu_{i 1}, \ldots, \mu_{i s}\right)$ и дисперсиями $\left(\sigma_{i 1}, \ldots, \sigma_{i s}\right)$.

Непрерывные случайные величины, соответствующие узлам графа, обозначим через $Z_{1}$. Считаем, что каждая из этих величин имеет нормальное распределение с параметрами $\left(\mu^{*}\right.$, $\sigma^{*}{ }_{1}$, , где $l=1, \ldots, r$. Совместное влияние непрерывной случайной величины $Z_{1}$ и реализаций дискретных величин на результирующую случайную величину $Y$ характеризуется весовыми коэффициентами $k_{1, i 1}, \ldots, i_{s} l=1, \ldots r$.

Тогда характеристики результирующего узла $Y$ вычисляются по формулам [10]:

$$
\begin{gathered}
\mu=\sum_{i 1}^{n 1} \ldots \sum_{i s}^{n s} p_{1, i 1} \ldots p_{s, i s}\left(\mu_{i 1, \ldots, i s}+\sum_{l=1}^{r} K_{l, i 1, \ldots, i s} \times \mu_{l}\right), \\
\sigma=\sum_{i 1}^{n 1} \ldots \sum_{i s}^{n s} p_{1, i 1} \ldots p_{s, i s}\left(\left(\mu_{i 1, \ldots, i s}+\sum_{l=1}^{r} K_{l, i 1, \ldots, i s}\right)^{2}+\right. \\
\left.+\sigma_{i 1, \ldots, i s}+\sum_{l=1}^{r} K_{l, i 1, \ldots, i s}^{2} \times \sigma_{l}\right)-\mu^{2} .
\end{gathered}
$$

На рис. 4 результирующая непрерывная переменная У (урожайность зерновых) зависит от дискретной переменной - СУП $(s=1)$ и двух непрерывных переменных: $S$ и ОК $(r=2)$. Для У значения $\mu$ и $\sigma$ могут быть рассчитаны с учетом соответствующих параметров случайных величин: СУП, $S$ и ОК по формулам (2), (3). Также $\mu$ и $\sigma$ для У может быть рассчитано с помощью соответствующей автоматно-вероятностной модели, которая учитывает разные возможные реализации величин СУП, $S, \mathrm{OK}$ и др. 
После исследования возможного уровня урожайности для определенного вида зерновых анализируем процесс его обработки на зерносушильных установках.

На рис. 5 результирующая переменная $V$ зависит от дискретной переменной $R Z(s=2)$ и непрерывных переменных: АП и нормы амортизации: $N A(r=2)$.

Рассмотрим функционирование двух модулей зерносушильных устройств. Балансовая стоимость каждого составляет примерно 20000 у.е. $R A$ - расходы на амортизацию каждого модуля зависят от балансовой стоимости и нормы амортизации. Предположим, установленная норма амортизации (5-10 процентов от стоимости): $0,075 \pm 0,025$, т.е для вершины $N A \mu=0,075$, а $\sigma=(0,025)^{2}=0,000625$.

Также пусть ставка аренды одного га земли составляет в среднем 2000 у.е. и колеблется в пределах \pm 10 процентов, т.е. для вершины АП: $\mu=2000 ; \sigma=(200)^{2}=40000$ и площадь участков, закрепленных за каждым модулем, равна 0,5 га. Вершина $V$ - производственные затраты рассматривается как случайная величина, имеющая условно нормальное распределение на значениях родителей (вершины АП, $N A$ и $U Z$ ). Предположим, что уровень загрузки $U Z$ (дискретная переменная) для каждого из двух используемых модулей соответственно равен: 0,4 и 0,6. При расчете производственных затрат следует включать амортизационные расходы $R A$ по каждому модулю, арендную плату за соответствующие площади и прямые затраты (ПЗ), связанные с поддержанием нормальной работы данных модулей. Согласно характеристике зерносушильных установок Vesta 5, с учетом использования дизельного топлива, ПЗ для первого модуля примем 5000 у.е., а для второго 5200 у.е., причем точность расчетов соответствует пяти процентам.

Учитывая параметры распределения вершин АП, $N A, U Z$, для вершины $V$ в соответствии с формулами (2), (3) рассчитываем значения параметров $\mu$ и $\sigma$ (табл. 4):

$$
\begin{gathered}
\mu=\sum_{i=1}^{2} p_{i}\left(\mu_{i}+\sum_{l=1}^{2} K_{l, i} \times \mu_{l}\right)= \\
=0,4 \times(5000+20000 \times 0,075+
\end{gathered}
$$

$$
\begin{gathered}
+0,5 \times 2000)+0,6(5200+20000 \times 0,075+ \\
+0,5 \times 2000)=3000+4620=7620, \\
\sigma=\sum_{i=1}^{2} p_{i}\left(\left(\mu_{i}+\sum_{l=1}^{2} K_{l, i} \times \mu_{l}\right)^{2}+\sigma_{i}+\right. \\
\left.+\sum_{l=1}^{2} K_{l, i}^{2} \times \sigma_{l}\right)-\mu^{2}=656100, \sqrt{\sigma}=810 .
\end{gathered}
$$

Например, при анализе производственных затрат $V$ (рис. 5) и осуществлении расчета параметров закона распределения данной случайной величины использовалась соответствующая входная информация (табл. 2, 3) и проводились необходимые расчеты по формулам (2), (3), которые определяют получение выходной информации (табл. 4).

Т а б ли ц а 2

\begin{tabular}{|c|c|}
\hline \multicolumn{2}{|c|}{ Арендная плата } \\
\hline$\mu$ & 2000 \\
\hline$\sigma$ & $40000=200 \times 200$ \\
\hline
\end{tabular}

Т а бли ц а 3

\begin{tabular}{|c|c|}
\hline \multicolumn{2}{|c|}{ Норма амортизации } \\
\hline$\mu$ & 0,075 \\
\hline$\sigma$ & $(0,025)^{2}$ \\
\hline
\end{tabular}

Т а б ли ц а 4

\begin{tabular}{|c|c|}
\hline \multicolumn{2}{|c|}{ Производственные затраты } \\
\hline$\mu$ & 7620 \\
\hline$\sigma$ & 656100 \\
\hline
\end{tabular}

На рис. 6 результирующая переменная $R$ исследуется в зависимости от влияния двух непрерывных переменных: У - урожайность и $V-$ затраты $(r=2)$, параметры которых предварительно рассчитаны. Определив значения $\mu$ и $\sigma$ по формулам (2) и (3) для случайной величины $R$, рассчитываем с помощью таблиц нормального распределения значения (табл. 5):

$$
P\left(x^{*} \leq R<x^{* *}\right)=\int_{x^{*}}^{x^{*}} e^{-\frac{(z-\mu)^{2}}{2 \sigma^{2}}} d z
$$

где значения $x^{*}$ и $x^{* *}$ случайной величины $R$ соответствуют границам определенного интервала устойчивости производства и задаются экспертами.

Т а б ли ц а 5

\begin{tabular}{|c|c|}
\hline$\mu$ & 43,3 \\
\hline$\sigma$ & 11,41 \\
\hline$X^{*}$ & 40 \\
\hline$X^{* *}$ & 45 \\
\hline$P\left(x^{*} \leq R<x^{* *}\right)$ & 0,87 \\
\hline
\end{tabular}


Разработанная система моделей позволяет для избранных сценариев развития группы предприятий отрасли растениеводства, соответствующих определенным стратегиям внедрения инновационно-инвестиционных технологий, осуществлять ранжирование предприятий отрасли растениеводства по значениям показателей, характеризующих экономическую устойчивость отрасли.

Заключение. Перспективные направления развития интеллектуальных информационных систем управления - разработки, связанные с представлением, приобретением, пополнением, манипулированием, обобщением знаний, а также поиском путей классификации, формализации знаний и схем рассуждений и умозаключений. Интеллектуализация информационных систем предусматривает возможность предложения управленческих решений на основе знаний, наполняемых базу знаний. В ходе исследования обоснована целесообразность построения и использования БСД как ЭС при анализе экономической эффективности функционирования группы предприятий отрасли.

Постепенная адаптация и уточнение модели осуществляется благодаря расчету и перерасчету тензора условных вероятностей исследуемых показателей, характеризующих экономическую устойчивость отрасли.

Это будет способствовать накоплению и уточнению определенных знаний, что в свою очередь позволяет уточнять расчеты определенных необходимых экономических и экологических показателей, рассчитываемых с помощью автоматно-вероятностной модели исследования процесса миграции и накопления веществ (тяжелых металлов и др.) в почве определенного типа при определении урожайности сельскохозяйственных культур [9]. Данный подход лежит в основе методологии ранжирования предпри- ятий отрасли по значениям показателей, характеризующих экономическую устойчивость.

1. Бутаев Ш. Интеллектуализация процессов принятия решений в информационных системах управления. - http://infocom.uz/2008/05/09/intellektualizatsiya-protsessov-prinyatiya-resheniy-v-informatsionnyih-sistemah-upravleniyal

2. Бідюк П.І. Оцінювання і прогнозування стану малого підприємства за допомогою мережі Байєса // Наук. пр. Миколаївського дер. гум. ун-ту ім. Петра Могили, 2005. - 44. - С. 7-29.

3. Бідюк П.І., Кожухівський А.Д., Кожухівська О.А. Система підтримки прийняття рішень для аналізу i прогнозування стану підприємства // Прогресивні інформаційні технології. Радіоелектроніка, інформатика, управління. ЗНТУ. - 2013. - № 1. - С. 128-136.

4. Pearl J. Probabilistic Reasoning in Intelligent Systems: Networks of Plausible Inference / J. Pearl-San Mateo, CA (USA): Morgan Kauffmann Publ., Inc., 1988. - 550 p.

5. Згуровський М.3., Бідюк П.І., Терентьєв О.М. Системна методика побудови Байєсовських мереж // Наукові вісті НТУУ «КПІ». - 2007. - № 4. - С. 47-61.

6. Системи підтримки прийняття рішень - проектування та реалізація / П.І. Бідюк, Ю.Ю. Щербань, В.Ю. Щербань та ін.; Київськ. нац. ун-т технологій та дизайну. - К., 2004. - 112 с.

7. Соболевська Л.І. Принципові підходи до управління стійким розвитком аграрного сектора / Соболевська Л.І. // Матеріали школи-семінару «Перспективні технології прийняття рішень в умовах систем інтелектуального управління бізнесом» / Матеріали школи-семінару (Жукін, 30 червня - 5 липня 2014. - C. 184-194). - www.irtc.org.ua

8. Михалевич В.С., Шор Н.З. Метод последовательного анализа вариантов при решении вариационных задач управления планирования и проектирования // IV Всесоюзн. математический съезд. Л.: ЛГУ, 1961. - $91 \mathrm{c}$.

9. Соболевська Л.І., Цюпко С. Моделювання процесу внесення хімічних речовин у грунт певного типу // Економіст. - 2005. - № 2. - С. 71-73.

10. Бидюк П.И., Терентьев А.Н., Гасанов А.С. Построение и методы обучения Байесовских сетей // Кибернетика и системный анализ. - 2005. - № 4. C. $133-147$.

Тел. для справок: +38 044 285-6202 (Киев) (С) Л.И. Соболевская, 2016 


\section{L.I. Sobolevskaya}

The Approach to the Intellectualization of the Decision Support Systems in the Evaluation and Analysis of the Plant Growing Enterprises

Keywords: agrarian sector, expert systems, Bayesian approach, Bayesian Networks.

Expert systems (ES) are regarded as as type of system for the support of decision-making processes which allows using knowledge and expertise accumulated by the specialsts to generate new knowledge.

The aim of the research is to define the main principles of the development of a knowledge-oriented model taking into consideration the factors necessary for the stable development of the plant growing branch.

The research proves expediency of constructing and using the Bayesian Belief Networks as an expert system (ES) for analysing the economical effectiveness of plant growing enterprises.

For calculation of the certain indices it is offered to construct and use the machine and probabilistic model for the research of the material migration and accumulation in a particular soil type when estimating the crop capacity of agricultural plants.

The developed model systems allows to rang the enterprises according to the indices characterising the economical stability of the branch.

Intellectualization of the decision-making processes in the framework of modern informational executive systems facilitates the effectiveness of the managerial decision-making. 